\title{
The Posterior Parietal Cortex Encodes in Parallel Both Goals for Double-Reach Sequences
}

\author{
Daniel Baldauf, He Cui, and Richard A. Andersen \\ Division of Biology, California Institute of Technology, Pasadena, California 91125
}

The parietal reach region (PRR) is known to be involved in the preparation of visually guided arm movements to single targets. We explored whether PRR encodes only the target of the next movement or, alternatively, also a subsequent goal in a double-reach sequence. Two monkeys were trained to memorize the locations of two peripheral cues and to prepare for a memory-guided delayed double-reach sequence. $\mathrm{On} \mathrm{a} \mathrm{GO}$-signal they had to reach in a predefined order to both remembered target locations without breaking eye fixation. The movement goals were arranged such that either the first or the second target was inside the response field of an isolated neuron. We analyzed the neural activity of single cells in PRR during the late memory period between cue offset and the GO-signal. During this memory period, most PRR cells encoded the first as well as the second goal of the planned reaching sequence. The results indicate that the posterior parietal cortex is involved in the spatial planning of more complex action patterns and represents immediate and subsequent movement goals.

Key words: double-reach; motor planning; parietal reach region; movement sequences; sensorimotor; hand; attention

\section{Introduction}

Many actions we perform every day are complex, coordinated concatenations of single movements. Movement sequences are crucial to perform fluently in various tasks. Several studies have started to investigate how the primate brain programs sequential movements. It has been shown that frontal circuits, especially prefrontal areas (Averbeck et al., 2006; Mushiake et al., 2006; Shima et al., 2007), primary motor (Lu and Ashe, 2005), premotor (Ohbayashi et al., 2003), and supplementary motor areas (Tanji and Shima, 1994), store information that is essential to execute a sequential movement. These frontal areas seem to be involved in the encoding of subsequent movement parts, their directions, and the temporal organization of the sequence (Shima and Tanji, 1998; Fujii and Graybiel, 2003; Ninokura et al., 2003; Histed and Miller, 2006).

However, the preparation for a movement sequence to multiple targets certainly involves other brain regions. For example, visual cortex processes the targets that guide the motor sequence. It has been shown that the intention to sequentially reach to various goal locations leads to preferential visual processing at all of these distinct target zones, well before the motor sequence actually starts (Baldauf et al., 2006; Baldauf and Deubel, 2008a,b). These sensory facilitations appear to arise from attentional topdown signals that are back-projected from motor planning areas.

\footnotetext{
Received July 20, 2008; accepted Aug. 11, 2008.

This work was supported by the German Academic Exchange Service (Deutscher Akademischer Austausch Dienst), the Fulbright Commission, the National Eye Institute, and the James G. Boswell Foundation. We thank Tessa Yao for editorial assistance, Kelsie Pejsa and Nicole Sammons for animal care, EunJung Hwang and Viktor Shcherbatyuk for technical assistance, and Heiner Deubel.

Correspondence should be addressed to Daniel Baldauf, Division of Biology, Mail Code 216-76, California Institute of Technology, Pasadena, CA 91125.E-mail: daniel@vis.caltech.edu. DOI:10.1523/JNEUROSCI.3423-08.2008

Copyright $\odot 2008$ Society for Neuroscience $\quad$ 0270-6474/08/2810081-09\$15.00/0
}

The posterior parietal cortex (PPC), known as a sensorimotor interface, transforms the visual information about a target object into movement intentions (Andersen and Buneo, 2002; Buneo and Andersen, 2006). A subdivision of PPC, the parietal reach region (PRR), was found to be involved in arm movements (Snyder et al., 1997; Cui and Andersen, 2007) and guide the target selection for an impending reach (Scherberger and Andersen, 2007). So far, however, there has not been found any evidence for the representation of subsequent hand movement goals in parietal cortex. This is remarkable because one of the functions of the parietal cortex is thought to be the allocation of attention (Colby and Goldberg, 1999; Rushworth et al., 2001; Corbetta and Shulman, 2002; Bisley and Goldberg, 2003). It is therefore a very likely source of top-down signals that cause attentional modulation at all goal locations in a movement sequence. Indeed, recent functional MRI studies in humans showed that PPC has the capacity to store multiple relevant targets in a match-to-sample task (Todd and Marois, 2004) and that it is involved in the serial organization of motor behavior (Jubault et al., 2007). Furthermore, the blood oxygenation level-dependent activity in PPC depended on the number of targets that had to be memorized for a delayed sequence of saccades (Medendorp et al., 2006). In a previous study, Batista and Andersen (2001) recorded single-cell activity in the PRR while monkeys performed double reaches to sequentially presented targets but did not find a representation of subsequent movement goals. Here, we readdress the encoding of multiple movement goals in PPC with a different sequential reaching task in which two targets were presented in parallel (simultaneously).

\section{Materials and Methods}

We recorded the activity of single neurons in PRR while monkeys prepared for sequential double-reaches to two peripheral locations. To dis- 
sociate between planning activity related to the first versus the second sequence component, we arranged the targets such that either the goal for the first or the goal for the second reach was inside the response field of a neuron.

Animal preparation. Both monkeys (Macaca mulatta) were implanted with a head holder and a recording chamber above the intraparietal sulcus in the posterior parietal cortex. The surgeries were conducted under inhaled anesthesia. All surgical procedures and the animal care protocols were approved by the California Institute of Technology Animal Care and Use Committee and in accordance with the $\mathrm{Na}$ tional Institutes of Health guidelines.

Recordings. During the experiment the headfixed animal sat in a dark room in front of a touch-sensitive screen. Viewing and reaching distance was $31 \mathrm{~cm}$. Eye movements were recorded with a video-based infrared eye tracker (ISCAN). A microelectrode array was inserted into the cortex beneath the recording chamber using a five-channel micro drive (Thomas Recording). The device allowed for simultaneous extracellular recordings from up to five microelectrodes (single-core Quartzglas-platinum/ tungsten electrodes). The raw signals from each electrode were preamplified, digitalized, and spike-sorted online (Sort Client; Plexon) to isolate single neurons. The quality of the online sorting procedure was later controlled with an Offline Sorter (Plexon). In both monkeys we recorded in a $3 \times 3 \mathrm{~mm}$ region of the chamber, typically 3-6 mm below the dura. Structural magnetic resonance imaging (MRI) was used to identify the position of the intraparietal sulcus for placement of the recording chambers and electrode penetrations (Fig. $1 \mathrm{~A}$ ), and the recording site was defined as PRR based on the functional criterion of higher planning activity for reaches than for saccades.

Behavioral tasks. To map the receptive field of an isolated neuron, the animal was first requested to perform a memory-guided singlereach $(\mathrm{SR})$ task (Fig. $1 \mathrm{~B}$ ). At the beginning of each trial, a fixation point (red square) was presented at the center of the screen, accompanied by a green circle for initial hand positioning (radius $0.9^{\circ}$ ). The monkey had to fixate the central red box throughout the entire trial. Deviations of eye position bigger than $3^{\circ}$ caused the program to abort the trial. After the monkey positioned his hand for 500-600 ms at the center, the trial was initialized and a green square was presented at $17^{\circ}(9.1 \mathrm{~cm})$ eccentricity, indicating the goal location for a subsequent reach. This reach target was presented for $400 \mathrm{~ms}$ at one of eight possible locations that equidistantly surrounded the center. After the reach target was extinguished, a memory period ensued and the monkey had to keep his hand at the initial hand position for another $600 \mathrm{~ms}$. At the end of this memory period, a GO-signal was given by extinguishing the central green hand fixation point. Then the monkey had to reach for the previously cued location without breaking eye fixation. If within $600 \mathrm{~ms}$ the monkey reached into a tolerance window that surrounded the target location by $4^{\circ}$ of visual angle, the target reappeared at its original position and the monkey was rewarded with juice. Typically, we recorded two blocks of single reaches (one before and one after the double-reach block) with five trials for each of the eight directions.
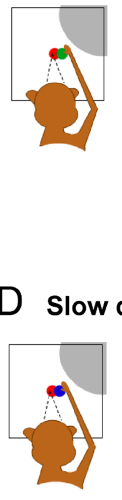

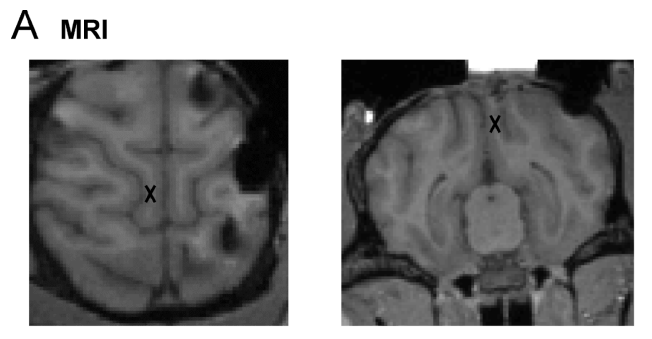

\section{B Single-reach task (SR)}

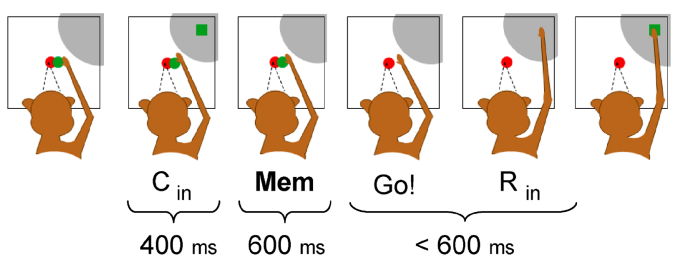

C Rapid double-reach task (rDR)
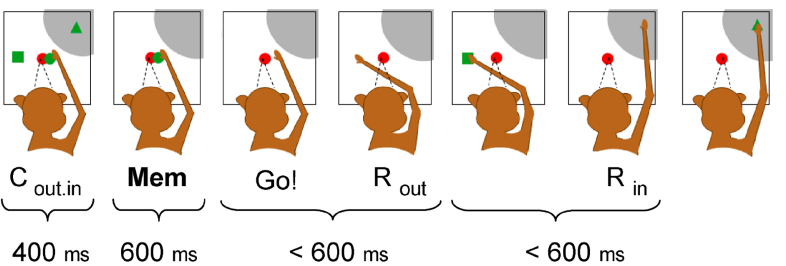

D Slow double-reach task (sDR)
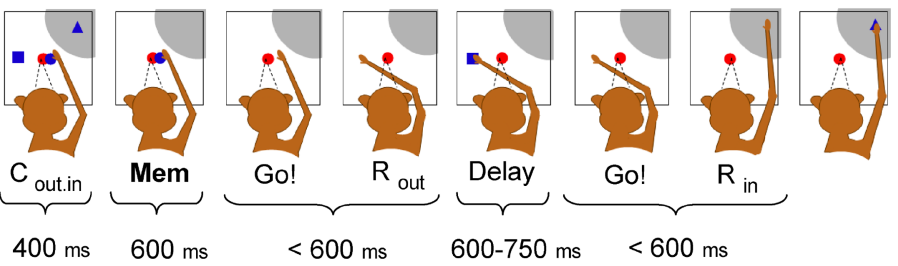

in

Figure 1. Localization of the recording site and the behavioral tasks. $A$, Localization of the recording sites in PRR in the medial bank of the intraparietal sulcus in monkey Z (left, axial; right, coronal). $\boldsymbol{B}-\boldsymbol{D}$, The sequence of stimuli and the monkeys' behavior in the various experimental tasks. Each panel shows a sketch of the monkey sitting in front of the touch-screen. Below the panels are the labels of the task epochs and their durations. The gray shadow indicates the response field of an example cell. In all variants of the task the monkey had to keep his eyes fixated at the central red square throughout the whole trial. $\boldsymbol{B}$, The delayed single-reach task was used to map the response field of an isolated neuron. At the beginning of each trial the monkey positioned his hand at the central green circle. During the cue period $\left(C_{\text {in }}\right)$, a peripheral green square cued a movement goal for $400 \mathrm{~ms}$. After the cue was extinguished, the monkey had to memorize this location for $600 \mathrm{~ms}$ (Mem) until the central green circle disappeared (G0-signal, Go!). The monkey then had to reach to the cued location within $600 \mathrm{~ms}$. After the reach was completed, a $100 \mathrm{~ms}$ flash of the green circle at its original position provided visual feedback about the accuracy and the monkey received a juice reward. $C$, In the rapid double-reach task a first and second reach goal were simultaneously cued by a green square and triangle, respectively. After the memory period, the monkey had to reach first to the location in which the square had been presented and then to the location in which the triangle had been shown. Both reaches had to be performed in a rapid sequence within $600 \mathrm{~ms}$ each. Once a position was reached with sufficient accuracy, the original cue stimulus flashed again for $100 \mathrm{~ms}$. $\boldsymbol{D}$, The slow double-reach task was identical to the rapid version except that a $600-750$ ms delay ensued between the first and second reach (Delay). After the monkey reached to the first goal, the blue square reappeared for $600-750 \mathrm{~ms}$ and the animal had to hold his hand at the reached location until this stimulus disappeared again (second $\mathrm{GO}$-signal, Go!). After this second $\mathrm{GO}$-signal was provided, the monkey continued the sequence production.

After the response field of a cell was identified, the monkey performed the rapid double-reach task (rDR) (Fig. 1C), which is structured similarly to the single-reach task. Here two targets were simultaneously presented to the monkey during the $400 \mathrm{~ms}$ cue period: a green square indicated the goal for the first reach and a green triangle the goal for the second reach. In each trial, the position of the goal for the first reach was randomly chosen from the eight potential goal locations. The second target, however, always appeared (at the same eccentricity) shifted by $135^{\circ}$ in a clockwise direction. A separation of $135^{\circ}$ was typically sufficient to allow us to present one target inside and the other outside the response field of 
a PRR cell. As in the SR trials, the cues were presented for $400 \mathrm{~ms}$. Then both cues were extinguished and the monkey had to keep his hand at the central position and remember both target locations. After another 600 $\mathrm{ms}$, the central green dot disappeared (GO-signal) and the monkey was allowed to start with the movement sequence. He had to first reach to the location in which the green square had been and then move on to the location in which the green triangle had been presented. The sequence production was rapid. Each reach had to be completed within $600 \mathrm{~ms}$, so the final goal location had to be reached no later than $1200 \mathrm{~ms}$ after the GO-signal. Otherwise the trial was aborted and no reward was given. The spatial tolerance at both goal locations was the same as in SR trials $\left(4^{\circ}\right.$ of visual angle). Again, we provided feedback about the accuracy of the first and second reach component. Therefore, the first and second target reappeared for $100 \mathrm{~ms}$ as soon as the monkey reached inside the respective tolerance window. Typically, we recorded 10 trials of these double-reach sequences for each direction.

A subset of all recorded cells ( 24 in monkey $\mathrm{C}$ and 12 in monkey Z) was further tested in an additional slow double-reach condition (sDR) (Fig. $1 D$ ), in which the first and second reaches were interrupted by an interreach delay. In these trials a blue circle (next to the red square for eye fixation) marked the central hand fixation, and the first and second reach goals were cued by a blue square and a blue triangle, respectively. After the cueing phase, the targets again had to be remembered for $600 \mathrm{~ms}$ and the extinction of the central hand fixation served as the first GO-signal. The monkey was trained to reach to the first target location within 600 ms, causing the initial cue to reappear as feedback stimulus. Without breaking eye fixation, he now had to keep his hand close to the reappeared first movement goal until it disappeared again, signaling a second GO-signal. Once the feedback stimulus disappeared, the monkey had to reach within $600 \mathrm{~ms}$ to the second goal location, at which he got a final feedback about accuracy (reappearing of the second cue) and reward. During the delay between the first and second reach, the monkey usually put his hand just to the side of the reappeared stimulus (but still within the tolerance window around the goal) to not occlude the second GOsignal. The inter-reach delay was randomized between 600 and $750 \mathrm{~ms}$. At the very beginning of each block the monkey knew by the color of the central hand fixation whether he had to perform an rDR or an sDR. Rapid and slow blocks were alternated.

After the block with DR trials was completed, another block of 40 SR trials ( 5 trials $\times 8$ directions) was recorded from the same isolated cell to ensure that the response field of the cell did not change during the recording session.

Data analysis. We analyzed the neural activity during the last $400 \mathrm{~ms}$ of the memory period. Only cells with significant spatial tuning during this memory period of the SR task were further tested and analyzed under the double-reach condition. For the analysis of the double reach trials, we compared the memory activity of a cell in three different sequence conditions that are of special interest for the purpose of the study: (1) in the condition $\mathrm{DR}_{\text {in.out }}$, only the first target is inside of the response field of the cell and the second is outside (Fig. 2B); (2) in condition $\mathrm{DR}_{\text {out.in }}$, in contrast, the goal for the second reach is inside the response field but the first target is not; finally, (3) in condition $\mathrm{DR}_{\text {out.out }}$, both the first and second reaches are directed to locations that lie outside the response field of the cell. The activity during this last sequence type, $\mathrm{DR}_{\text {out.out }}$, serves as a baseline. To quantitatively describe the planning activity of a cell, an index was computed:

$$
\begin{aligned}
\text { Index }=\left(\mathrm{DR}_{\text {out.in }}-\mathrm{DR}_{\text {out.out }}\right) /\left[\left(\mathrm{DR}_{\text {out.in }}\right.\right. & \left.-\mathrm{DR}_{\text {out.out }}\right) \\
& \left.+\left(\mathrm{DR}_{\text {in.out }}-\mathrm{DR}_{\text {out.out }}\right)\right],
\end{aligned}
$$

where $\mathrm{DR}_{\text {out.in }}, \mathrm{DR}_{\text {in.out }}$, and $\mathrm{DR}_{\text {out.out }}$ refer to the mean firing rates during the memory periods before the respective type of double-reach sequence. The index relates the mean memory activity in $\mathrm{DR}_{\text {out.in }}$ to the sum of the mean activities in $\mathrm{DR}_{\text {in.out }}$ and $\mathrm{DR}_{\text {out.in }}$, each after subtracting the baseline activity, i.e., the mean of $\mathrm{DR}_{\text {out.out }}$ A positive index value indicates that the mean firing rate in $\mathrm{DR}_{\text {out.in }}$ trials is higher than in $\mathrm{DR}_{\text {out.out }}$, and therefore, the cell does also encode the second reach. On the contrary, index is 0 or even negative if the cell does not at all encode for the second reach goal but only for the first one. The index is 0.5 if the first and second goals are equally encoded. Indices between 0 and 0.5 occur for cells that code for both the first and the second reach goal but with a bias to stronger code for the first (immediate) goal. If the cell is more responsive to the second reach destination than to the first goal, the index will exceed a value of 0.5 . Cells that exclusively encode the second, final reach goal have values close to 1 .

Mathematically, the index of a cell could become erroneously positive if both memory activities in $\mathrm{DR}_{\text {in.out }}$ and $\mathrm{DR}_{\text {out.in }}$ trials were, on average, smaller than the memory activity of the cell in $\mathrm{DR}_{\text {out.out }}$ trials. This would mean that the response field of the cell had changed dramatically in DR trials. Therefore, we verified for each recorded cell that this is not the case and that the index of a cell is never spuriously positive.

\section{Results}

\section{Neuronal encoding of rapid double-reach sequences}

A total number of 112 neurons were recorded ( 70 in monkey $C$ and 42 in monkey $Z$ ) in the rapid double-reach condition. The results in both animals were qualitatively the same and will be jointly presented in the following results. Figure 2 shows three typical neurons examined in the single-reach and rapid doublereach task.

Under the SR condition (Fig. 2A), all three cells show strong spatial tuning. The appearance of the cue inside the response field of the cell elevated the firing rate. This activity stayed high throughout the whole memory period until the movement was finally initiated. A reach target presented outside the response field triggered little response. In the double-reach task three spatial arrangements of the two targets were of special interest: (1) the first goal was inside the response field of the cell but the second one was outside (condition $\mathrm{DR}_{\text {in.out }}$ ), or (2) only the second goal was inside the response field $\left(\mathrm{DR}_{\text {out.in }}\right)$, or (3) both targets lay outside the response field (baseline condition, $\mathrm{DR}_{\text {out.out }}$ ). Before a double-reach sequence (Fig. $2 \mathrm{~B}$ ), example cell 1 (first column) was activated during the memory period irrespective of whether the first or second target was in the receptive field of the cell. However, the firing rate did not increase if both reach goals were outside the response field. The second example cell (middle column) shows an elevated firing rate if the first reach goal is inside the response field, but only modest response if the subsequent goal is in the preferred direction (the difference in the mean firing rate is significant, $p<0.01$; Wilcoxon rank-sum test). The third example cell (right column) is more strongly activated if the second goal is in the response field $(p<0.05)$.

To quantify whether a cell showed a stronger representation for the first or second goal, we assigned an index to each cell that basically describes its activity during the memory period of $\mathrm{DR}_{\text {in.out }}$ versus $\mathrm{DR}_{\text {out.in }}$ trials (after subtracting the baseline memory activity, $\mathrm{DR}_{\text {out.out }}$ ) (see Materials and Methods). For a majority of cells, the indices range between 0 and 1 and cluster around the value 0.5 (mean $=0.47, p>0.3$, two-tailed $t$ test) (Fig. $3 A)$, indicating that for most of the neurons the memory activity was about the same whether the first or second reach goal was inside the response field, with a slight bias toward the representation of the first goal. In some neurons (like the second example cell), the mean memory activity in $\mathrm{DR}_{\text {out.in }}$ trials was close to the firing rate in $\mathrm{DR}_{\text {out.out }}$ trials (baseline) with resulting indices close to 0 . These cells only coded for the first goal, not for the second. However, we recorded some cells (like the third example cell) with indices close to 1 . These cells mainly coded for the second goal, not the first.

At the population level, the majority of neurons (75\%; $74 \%$ in monkey $\mathrm{C}$ and $77 \%$ in monkey $\mathrm{Z}$ ) were significantly more 


\section{A single-reach task (SR)}

Cell 1

Cue Mem
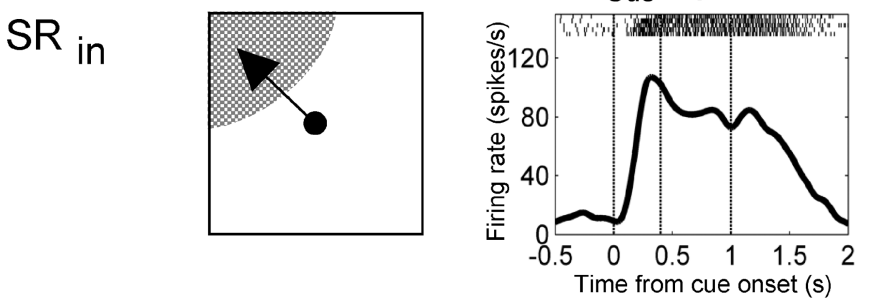

SR out

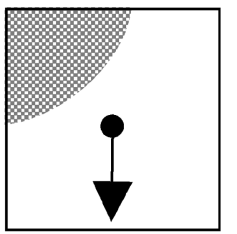

Cell 2
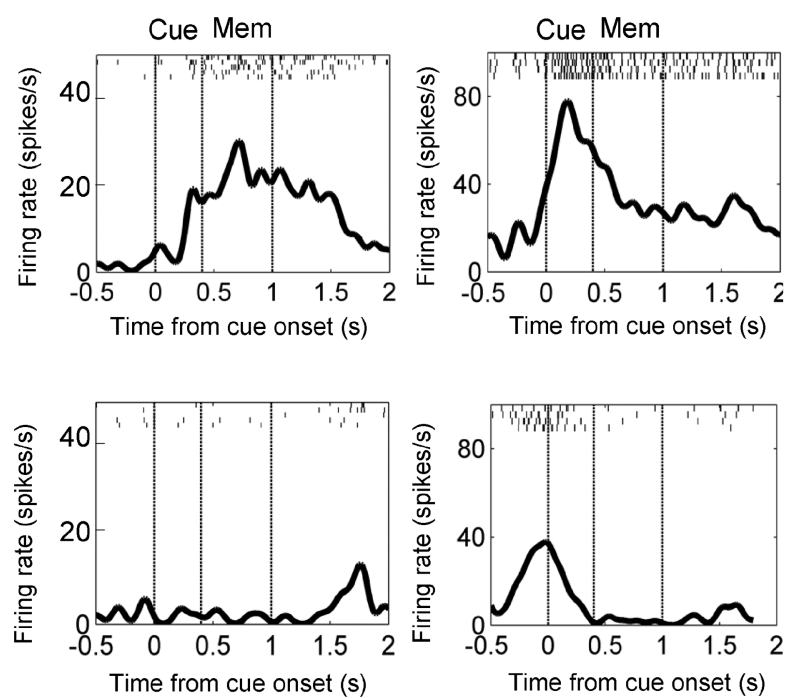

\section{B Rapid double-reach task (rDR)}
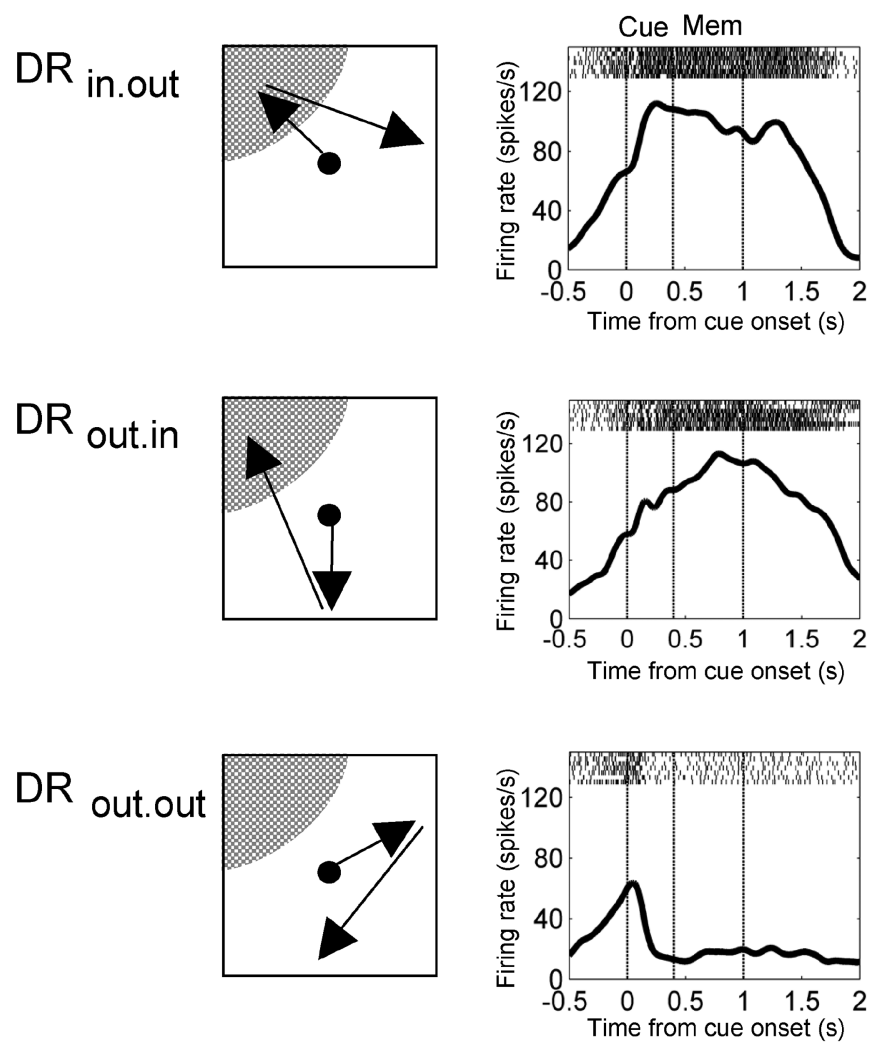
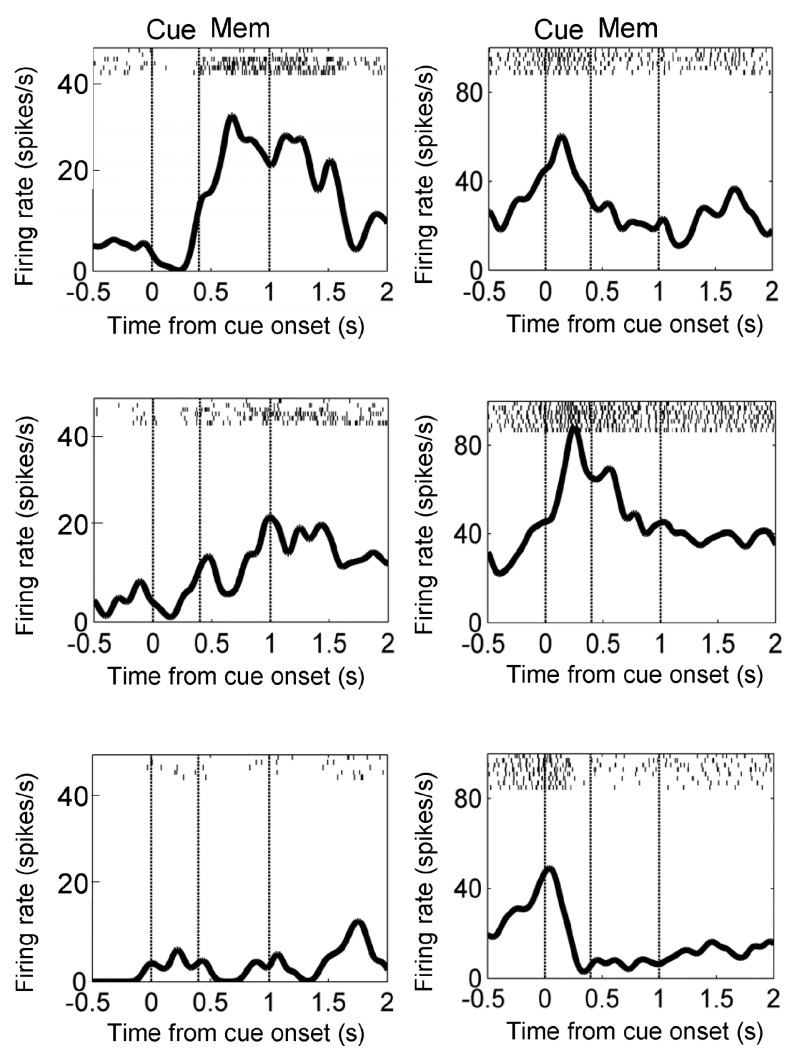

Figure 2. Response of three typical cells in posterior parietal cortex. The five rows show the average spiking activity of three example cells (columns 1-3) during various crucial task conditions. In each subplot, vertical dashed lines indicate, from left to right: cue onset $(0 \mathrm{~s})$, cue offset $(0.4 \mathrm{~s})$, and $\mathrm{G} 0$-signal $(1.0 \mathrm{~s})$. At the very top of each diagram the spike rasters are shown. During the memory period, i.e., between cue offset and $\mathrm{GO}$-signal, no visual stimulus was presented in the cells' response field. $\boldsymbol{A}$, Activity during the single-reach task when the goal was cued either inside (SR $\mathrm{in}_{\text {in }}$ first row) or outside ( $S R_{\text {out }}$ second row) the response fields of the cells. $\boldsymbol{B}$, The neurons' responses during the rapid double-reach task when either the first movement goal was inside the response field $\left(D R_{\text {in.out }}\right.$ third row), or the second ( $D R_{\text {out.in, }}$ forth row), or neither of them ( $D R_{\text {out out }}$ fifth row). The first example neuron (Cell 1 , first column) showed strong memory activity for the first and second goal and had an index of 0.51 (Fig. 3A) (see Materials and Methods). Example cell 2 (middle column) and cell 3 (right column) were selectively activated only if the first or second goal, respectively, was in the preferred direction. 
A

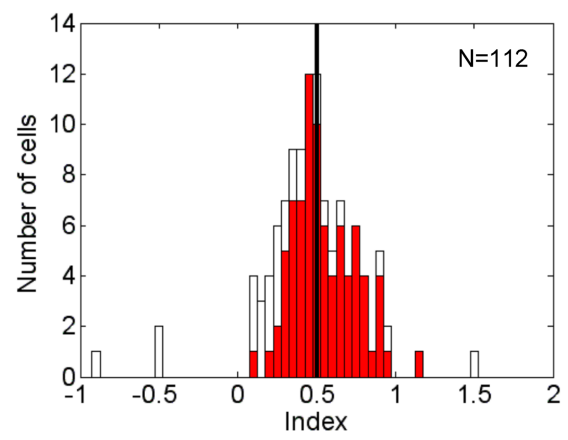

B

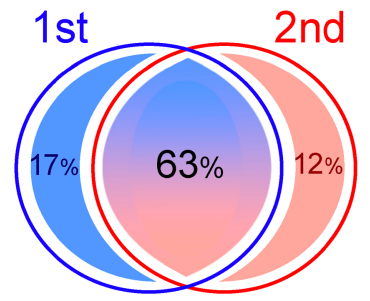

C

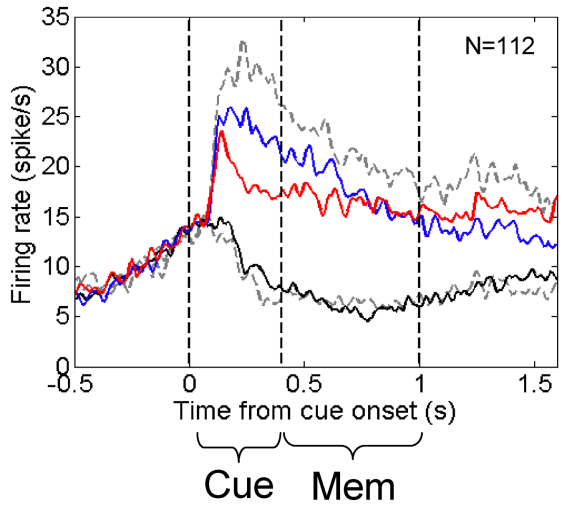

Figure 3. Population analysis. $\boldsymbol{A}$, Histogram of indices (see Materials and Methods) for the population of neurons in posterior parietal cortex tested in the rapid double-reach task. An index value close to 0 indicates that the cell encodes only the first reach goal during the late memory period, but not the second one. A positive index value indicates that the mean firing rate in $D R_{\text {out.in }}$ trials is higher than in $\mathrm{DR}_{\text {out.out }}$ and, therefore, the cell also encodes the second reach. The index is 0.5 (black vertical line) if the first and second goals are encoded to the very same extent. Indices between 0 and 0.5 occur for cells that code for both the first and the second reach goal, but with a bias to stronger code for the first (immediate) goal. If the cell is more responsive to the second (final) reach destination than to the first (intermediate) goal, the index will exceed a value of 0.5 . Cells that exclusively encode the second, final reach goal have values close to 1. Cells with significantly stronger activity for the second goal (DR out.in $_{\text {in }}$ than for the baseline condition $\left(D R_{\text {out.out }}\right.$ ) are marked in red; white bars show neurons without significant difference between $D R_{\text {out.in }}$ and $\mathrm{DR}_{\text {out.out }} \boldsymbol{B}$, Proportions of tested cells that showed significant elevated firing rates when only the first (blue circle) or second (red circle) goal or either goal (intersection) was in its receptive fields. $\boldsymbol{C}$, The population activity in the three double-reach conditions averaged over all 112 tested cells: $\mathrm{DR}_{\text {in.out }}$ (solid blue), $\mathrm{DR}_{\text {out.in }}$ (red), and $\mathrm{DR}_{\text {out.out }}$ (dashed black). The gray curves represent the average activity during single reaches into (dashed) and outside (dotted) the response field. Vertical lines mark the cue onset ( $0 \mathrm{~s})$, the cue offset $(0.4 \mathrm{~s})$, and the $\mathrm{GO}$-signal (i.e., the end of memory period, $1.0 \mathrm{~s}$ ).

strongly activated in $\mathrm{DR}_{\text {out.in }}$ trials than in $\mathrm{DR}_{\text {out.out }}$ trials and therefore significantly coded (also) for the second reach goal (Fig. $3 B$ ). Only small proportions of the tested population represented exclusively the first $(17 \% ; 18 \%$ in monkey $\mathrm{C}$ and $15 \%$ in monkey $\mathrm{Z})$ or the second goal $(12 \% ; 13 \%$ in monkey $\mathrm{C}$ and $7 \%$ in monkey $\mathrm{Z})$ in their response fields.

Figure $3 C$ shows the average response across all cells recorded in rapid double-reach sequences when either the first goal $\left(D_{\mathrm{in}^{-}}\right.$ .out, blue line) or the second ( $\mathrm{DR}_{\text {out.in }}$, red line) or neither $\left(\mathrm{DR}_{\mathrm{out}^{-}}\right.$ .out, black line) was inside the response field. For comparison, the average response in single-reach trials is drawn in gray. Most cells exhibited some anticipatory activity before cue onset. After a strong initial visual response to the cue (cue presentation from 0 to $400 \mathrm{~ms}$ ), there was persistently elevated activity throughout the memory period and even after the GO-signal, until the respective first or second reach was finally executed. In the baseline condition $\left(\mathrm{DR}_{\text {out.out }}\right)$, the initial anticipatory gain in firing rate disappeared even before the cuing period ended.

Previous studies (e.g., Batista et al., 1999) suggested that PRR encodes locations in an eye-centered frame of coordinates (i.e., with respect to the fovea). Alternatively, however, the representation of the second movement may be seen as the direction from the first target to the second (i.e., based on hand movement vectors). Therefore, we compared the planning activity of each cell for the second reach in eye-centered coordinates with the planning activity of the cell as if it were based on hand movement

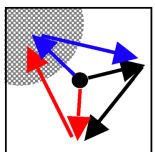

vectors. The eye-centered representation of a subsequent movement goal is the memory activity of the cell in such DR trials in which the second movement goal was placed inside the response field of the cell (as mapped in the SR task). The vector-based representation of the secondreach goal, however, is the memory activity of the cell in other DR trials, in which the second movement from the first to the second goal is directed in the preferred direction of the cell (as analyzed in the SR task). In our experimental paradigm, these two types of sequences differ by only one position, because both goals are always separated by $135^{\circ}$. Most of the cells (103 of 112 ) indeed showed a better representation of the subsequent movement goal in an eye-centered frame of reference. Only a minority of $8 \%$ of cells ( 9 of 112) better encoded the direction of the second reach component, i.e., the direction from the first goal to the second. This speaks in favor of an eye-centered representation of movement goals in PPC as reported previously. Note that for the first goal, the absolute position and movement direction are identical because the sequence always starts at the central fixation. The representation of the first movement goal is therefore unaffected by whether PPC neurons better encode the retinal position of a movement goal or the preferred direction of the hand movement.

\section{Neuronal encoding of slow double-reach sequences}

Monkeys might adopt different strategies to plan motor sequences during rapid and slow double-reaches. If there is a significant delay between two reaches, they may plan two movements one by one. In 36 neurons ( 24 neurons in monkey C, 12 neurons in monkey $\mathrm{Z}$ ) we directly compared the activity of cell during the memory period in rapid and slow double-reach sequences ( $r D R$ vs sDR). In the sDR sequences, the monkey prepared for interrupted, step-by-step sequences. In rapid sequences, the monkey's hand dwelled on the first goal location for only $240 \mathrm{~ms}$ on average ( $\mathrm{SD}=151 \mathrm{~ms}$ ) before continuing the second reach. In the slow version of the task, he had to wait at the first goal location for a randomized interval of 600-750 ms and then started the second reach with a latency of $298 \mathrm{~ms}$ (SD $=150$ $\mathrm{ms}$ ), resulting in an overall dwell time between both reaches of $970 \mathrm{~ms}$ on average. Figure 4 gives an example of one cell in monkey $\mathrm{C}$ under these two conditions of rapid versus slow sequence production. Also before interrupted sequences that required the monkey to pause after the first reach for a considerable delay (600 $\mathrm{ms}$ ) before continuing the sequence production, the second goal was encoded throughout the memory period.

Under slow sequence production, the planning activity of the late memory period was even stronger than that under rapidsequence production. In both conditions the planning activity only slightly, but continuously, decayed until the respective reach into the response field was executed. For the first reach goal, the decay rate was the same in fast versus slow sequence trials (Fig. 
$4 A$, top). However, the representation of the second goal (Fig. $4 A$, middle) decayed with a considerably slower rate under the slow versus under the rapid sequence condition ( $p<0.01$, Wilcoxon rank-sum test). As a consequence, in the slow version of the task, the representation of the second goal was activated longer, until the second sequence component was finally executed at the end of the second delay period.

The response-locked analysis of the firing rates (Fig. $4 \mathrm{~B}$ ) provides some further insights into the responsiveness of the neurons to visual stimuli. In all doublereach trials, the acquisition of the first reach goal was accompanied by visual feedback at the original cue location. Immediately after the first goal was reached, the very same visual stimulus that had been presented during the cue period reappeared at its original location (see Materials and Methods). In case of rapid sequences, the feedback was flashed for 100 $\mathrm{ms}$; in step-by-step sequences, however, the original stimulus reappeared for 600 ms. In the $\mathrm{DR}_{\text {in.out }}$ condition (Fig. $4 B$, top) the visual feedback was provided inside the receptive field of the cell. In the $\mathrm{DR}_{\text {out.in }}$ and $\mathrm{DR}_{\text {out.out }}$ conditions it reappeared outside the receptive field (Fig. $4 B$, middle and bottom). Most interestingly, the cell did not respond at all to this visual feedback stimulus in the receptive field (Fig. $4 B$, top) neither when it was briefly flashed (rDR, solid line) nor when it was presented for $>600 \mathrm{~ms}$ (sDR, dashed curve) and was behaviorally relevant to the task. When the feedback was given at a location outside the receptive field (Fig. $4 B$, middle) it did not distract the representation of the second goal inside the response field, i.e., it did not accelerate the decay of this representation.

On a population level (Fig. 5), it becomes apparent that the main difference in planning activity between slow and fast sequence production is in the representation of the second goal. Whereas the first goal representation is, on average, almost identical in both conditions (Fig. 5A, blue solid and blue dashed line for $\mathrm{DR}_{\text {in.out }}$ in rapid vs slow double reaches), the second goal representation is enhanced during the memory period before slow double sequences compared with rapid ones (Fig. 5A, dashed vs solid red curves). In rapid trials the first and second movement goals were represented to about the same extent, and there was no significant difference between the average memory activities in $\mathrm{DR}_{\text {in.out }}$ and $\mathrm{DR}_{\text {out.in }}(p>0.27, t(35)=-1.10)$. In the slow version, however, the second goal was significantly better encoded than the first one, i.e., the memory activity encoding the second reach goal was significantly higher than for the first goal $(p<0.05, t(35)=-2.95)$. This difference also becomes evident in the indices that were assigned to the individual cells. Figure $5 B$ illustrates the relation of each cell's index before rapid versus slow sequence production. Under rapid sequence conditions, the subset of 36 cells showed the same distribution of indices as the whole population (Fig. $3 A ; 112$ cells): clustering around
0.5 with a slight bias in support of the first movement goal (mean of $0.47,0.47$ in monkey C, 0.48 in monkey Z) (Fig. 5B, histogram given along the abscissa at the top). However, if the animal planned a step-by-step sequence (sDR), most of the cells changed their preferences toward a higher activity level for the secondary goal. This results in a shift of the index distribution toward 1 , with a mean of 0.58 ( 0.55 for monkey C, 0.63 for monkey Z) (Fig. $5 B$, histogram of indices under the slow sequence condition along the ordinate). A two-tailed $t$ test revealed the indices in slow versus rapid double reaches to be significantly different $(p<0.05)$. The main graph of Figure $5 B$ documents this change of preference for most of the neurons. Neurons with indices that are unaffected by whether a fast or slow sequence is planned are aligned along the diagonal.

We further analyzed the activities of the 36 cells while the sDRs were executed. During the second delay, i.e., the 600-750 ms interval after completion of the first reach but before the onset of the second movement, we directly compared the representation of the first and second movement goals. During this interval, the cells represented the second reach goal (which was still intended) more strongly compared with the first one, which was already accomplished ( $p=0.056, t(35)=-1.9766$ ) (see also Fig. S3, available at www.jneurosci.org as supplemental material). This is the case for most of the cells (70\%), although during this interval, the feedback stimulus is presented at the first goal location, and 
A
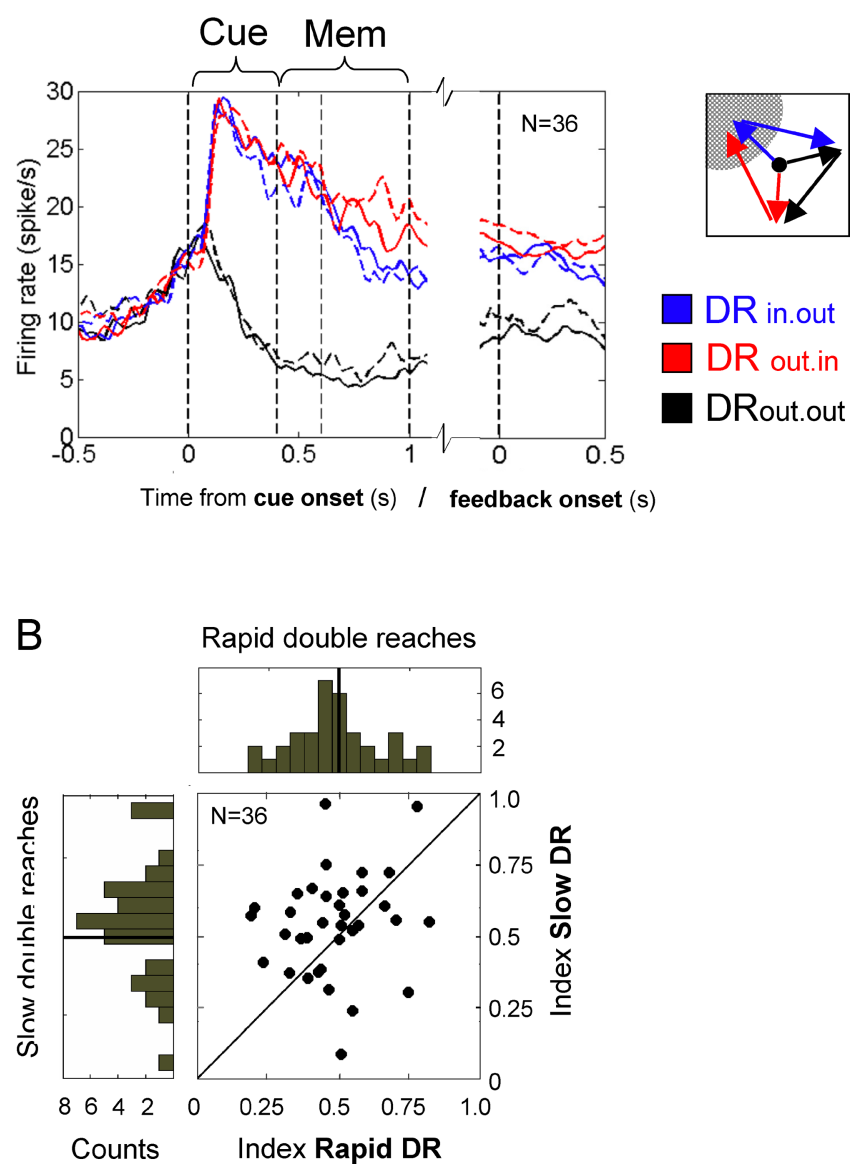

Figure 5. Population activity before slow versus rapid double reaches. $A$, Average activity of those 36 cells that were tested in the rapid (solid curves) versus slow (dashed curves) version of the double-reach task. The blue lines show population activity in trials in which the first goal is inside the cell's response field $\left(\mathrm{DR}_{\text {in.out }}\right)$. Red lines represent the average activity during trials in which only the second goal is inside the cell's preferred direction $\left(D R_{\text {out.in }}\right)$. The baseline, $D R_{\text {out.out }}$ is drawn in black. Vertical lines mark the cue onset $(0 \mathrm{~s})$, the cue offset $(0.4 \mathrm{~s})$, and the end of the memory period ( $\mathrm{GO}$-signal, $1.0 \mathrm{~s}$ ). On the left side, the neural activity is aligned to cue onset; on the right, it is aligned to the end of the first reach, i.e., feedback onset. Because of the relatively small size of the sample, the data are smoothed for presentation only. B, Comparison of indices (see Materials and Methods) that were computed for each of the 25 cells in the rapid (abscissa) versus slow (ordinate) double-reach condition. Most of the cells obtain a higher index before slow, interrupted sequence production than before rapid double reaches. The histograms of cell counts are given to the right and at the top (see Fig. 3A).

this could potentially add some sensory-driven activity to the representation of the first goal.

To further specify how the whole population encodes the information about the intended movement sequence, we applied a simple classification algorithm on a single-trial basis (see supplemental material, available at www.jneurosci.org). From the activation characteristics of all recorded cells during the preparation period, the algorithm successfully decoded the direction(s) of the intended reach(es) and whether it is going to be a single or double movement. This is additional evidence for a strong representation of the planned sequence (see supplemental material, available at www.jneurosci.org, for a more detailed discussion).

\section{Discussion}

In summary, our results suggest that PRR encodes in parallel the first and second goal for an intended double reach. The activation pattern in most of the cells is consistent with the hypothesis that
PRR simultaneously encodes the reach plans for subsequent reaches of an intended sequence. Multiple movement goals of the upcoming sequence are already represented before the first reach is started. The majority of single cells store the information about a goal that is in the cell's preferred direction independently of whether it is the first or the second component of the sequence.

Studies have demonstrated that several motor-cortical areas in the frontal lobe encode multiple goals during both target selection (Cisek and Kalaska 2005) and sequential movements (Tanji and Shima, 1994; Ohbayashi et al., 2003), suggesting that the visual information about multiple targets may be first encoded in the dorsal stream. Our results show that this is in fact the case; PRR encodes both goals for two sequential reaches.

\section{Differences from previous studies}

At first glance, our findings are partially in conflict with results from a previous study on the planning of multiple reaches in PRR (Batista and Andersen, 2001). Batista and Andersen (2001) showed that the parietal planning activity for a single goal location dropped when the reach plan shifted and an intervening reach to another location had to be executed first. They trained monkeys to prepare for a single reach to a memorized location. In some intervening trials, however, a further cue was presented on the opposite side, which the monkey also had to memorize. With onset of this second cue, the monkey had to cancel his original single-reach plan and now prepare for a double reach to the more recently cued location first, and only after another delay to continue with a second reach to the originally cued location. When the second cue appeared, the cells' activity for the original reach plan dropped significantly. The authors concluded that PRR does not code for subsequent reach goals.

It seems that the monkeys used different strategies to solve the tasks in both studies. In the paradigm of Batista and Andersen (2001), the monkeys planned one reach at a time, whereas in our task they simultaneously prepared for both reaches from the very beginning of each trial. Both tasks, however, differ in several important aspects and imply different strategic behavior.

First, we used a block design and provided the animal with strong context information about whether to plan single or double reaches. Especially the considerable amount of training in such a blocked design may allow the monkey to easily switch into different modes to be most efficient in both task variants. Second, in the paradigm of Batista and Andersen (2001), it is a central component that the original plan had to be cancelled. This may temporarily cause extra inhibition for the original goal location. Finally, the simple spatial arrangement of the second reach goal on the opposite side of the first one may not require preparation for a second goal-directed reach at all. The monkey may prepare for a simple center-out reach to the first goal and acquire the second goal by a rather unspecific backward movement that just overshoots the original starting position in the center. In contrast, the second reach requires a spatially exact preparation, if both targets are not center-symmetrically arranged.

\section{Attentional feedback signals}

One characteristic of the recorded parietal population is that $\sim 68 \%$ of cells had contralateral response fields, although always the ipsilateral hand was used for reaching. The output of the population that encodes the intention to reach to certain locations in eye-centered coordinates could be directly backprojected to cells in visual maps with congruent receptive fields.

Indeed, the observed population activity in $\mathrm{rDR}$ trials fits well with the results of a recent study on the distribution of visual 
attention during the preparation of rapid hand movement sequences (Baldauf et al., 2006) that reported attentional facilitation at multiple target locations before the initialization of speeded manual double sequences. The study showed that the ability to identify target letters was superior at all goal locations of a planned hand-movement sequence compared with other, movement-irrelevant positions. Similar to our results, the performance was greatest at the first reach goal and dropped with each subsequent goal position. In an event-related potential study, Baldauf and Deubel (2008b) showed that the preparation of a double reach enhances the processing of both movement goals in early visual areas. The tested PRR population is therefore a likely candidate [directly or indirectly via lateral intraparietal area (LIP)] to provide information about the task-relevance of action goals at certain locations and to back-project those top-down signals in an eye-centered frame of reference to visual areas to selectively facilitate visual perception at all goal locations.

This interpretation of PRR as specifically coding for reach goals is additionally supported by the lack of any response to the visual feedback at the end of the first movement (Fig. 4B). After the first goal was acquired, the initial visual stimulus reappeared as visual feedback. However, there was barely any response to the feedback stimulus. If the feedback was presented inside the response field, the activity did not rise, although the same stimulus elicited a massive response during the cue period. If the stimulus was presented outside the response field, the visual flash did not decrease the ongoing representation of the second goal, as one might suspect following a competition model (Desimone and Duncan, 1995). In both scenarios the neurons did not care about the appearance of the salient object, nor did it care that the animals had to covertly attend to the repeated offset of the feedback stimulus as it provided the second GO-signal in slow-sequence production trials. Once a stimulus lost its relevance as a movement goal after completion of the associated reach, its representation vanished, although it still provided task-relevant information about the accurate timing of the sequence production. This does not fit an interpretation of the parietal population as coding signals that are commonly referred to as "attention," in general. Cui and Andersen (2007) recorded cells in PRR and LIP while monkeys autonomously chose between saccading and reaching to acquire targets in the absence of direct instructions specifying which effector to use. They found complementary effector specificity of the two areas: PRR cells were selectively activated for reaches, whereas LIP cells were selective for saccade plans. If the monkey chose saccades, pre-movement activity in PRR dropped back to baseline level and did not show any enhancement related to general spatial attention. Therefore, it is likely that the PRR delay activity is largely related to limb-movement planning and not to a more general phenomenon such as attention.

This is in line with another study on PRR activity by Gail and Andersen (2006) that dissociated in an anti-reach task the cell responses to peripheral cues from the representation of reach targets. They showed that cells in PRR exhibited strong activity before anti-reaches if the reach goal was inside the cell's response field, but only rarely active if the visual cue was presented inside the field. These findings contrast with results in the eye movement-related homolog, area LIP, in which task-irrelevant distractors caused considerable activation during the cue and memory period (Platt and Glimcher, 1997; Bisley and Goldberg, 2003; Campos et al., 2006).

Instead of providing attentional signals per se, one possible interpretation of the recorded PPC activity is that there may be different attentional systems that provide top-down signals to sensory areas about different aspects of task relevance. The observed PRR population may contribute only those attentional top-down signals that specifically select movement targets for planned hand movements.

\section{Differential weighting of goal representations depending on sequence speed}

The analysis of trials with slow sequence production showed that there is also memory activity for the second goal if the sequence is interrupted. One conclusion that may be drawn is that the planning activity in parietal cortex incorporates even actions that take place in the more remote future. Furthermore, we recorded the same cells before slow and rapid sequences and found an increase in the representation of the second goal in slow trials. This finding mirrors the increased demands that are placed in the representation of the subsequent goals under slow sequence production: the movement plan for the second reach had to be kept active, not only during the memory period and the first reach, but also during another delay between both reaches. To compensate for the second reach plan to decay over time, and to expire before its execution, more activity was invested in its representation from the beginning. Under slow sequence production, the subsequent goal was assigned a higher priority compared with the immediate one because the risk to miss the correct location increases after such a prolonged delay without an additional spatial cue.

\section{References}

Andersen RA, Buneo CA (2002) Intentional maps in posterior parietal cortex. Annu Rev Neurosci 25:189-220.

Averbeck BB, Sohn JW, Lee D (2006) Activity in prefrontal cortex during dynamic selection of action sequences. Nat Neurosci 9:276-282.

Baldauf D, Deubel H (2008a) Properties of attentional selection during preparation of sequential saccades. Exp Brain Res 184:411-425.

Baldauf D, Deubel H (2008b) Attentional selection of multiple goal positions before rapid hand movement sequences: an event-related potential study. J Cogn Neurosci, in press.

Baldauf D, Wolf M, Deubel H (2006) Deployment of visual attention before sequences of goal-directed hand movements. Vision Res 46:4355-4374.

Balint R (1909) Seelenlaehmung des Schauens, optische Ataxie, raeumliche Stoerung der Aufmerksamkeit. Monatsschr Psychiatr Neurol 25:51-81.

Batista AP, Andersen RA (2001) The parietal reach region codes the next planned movement in a sequential reach task. J Neurophysiol 85:539-544.

Batista AP, Buneo CA, Snyder LH, Andersen RA (1999) Reach plans in eyecentered coordinates. Science 285:257-260.

Bisley JW, Goldberg ME (2003) Neural activity in LIP and spatial attention. Science 299:81-86.

Buneo CA, Andersen RA (2006) The posterior parietal cortex: sensorimotor interface for the planning and online control of visually guided movements. Neuropsychologia 44:2594-2606.

Buneo CA, Jarvis MR, Batista AP, Andersen RA (2002) Direct visuomotor transformations for reaching. Nature 416:632-636.

Campos M, Breznen B, Andersen RA (2006) Selection of targets and active disregard of irrelevant information in monkey LIP and SEF. Soc Neurosci Abstr 32:549.1.

Cisek P, Kalaska JF (2005) Neural correlates of reaching decisions in dorsal premotor cortex: specification of multiple direction choices and final selection of action. Neuron 45:801-814.

Colby CL, Goldberg ME (1999) Space and attention in parietal cortex. Annu Rev Neurosci 23:319-349.

Corbetta M, Shulman GL (2002) Control of goal-directed and stimulusdriven attention in the brain. Nat Rev Neurosci 3:201-215.

Cui H, Andersen RA (2007) Posterior parietal cortex encodes autonomously selected motor plans. Neuron 56:552-559.

Desimone R, Duncan J (1995) Neural mechanisms of selective visual attention. Annu Rev Neurosci 18:193-222.

Duda RO, Hart PE, Stork DG (2001) Pattern classification. New York: Wiley. 
Fujii N, Graybiel AM (2003) Representation of action sequence boundaries by macaque prefrontal cortical neurons. Science 301:1246-1249.

Gail A, Andersen RA (2006) Neural dynamics in monkey parietal reach region reflect context-specific sensorimotor transformations. J Neurosci 26(37):9376-9384.

Histed MH, Miller EK (2006) Microstimulation of frontal cortex can reorder a remembered spatial sequence. PLoS Biol 4:e134.

Jubault T, Ody C, Koechlin E (2007) Serial organization of human behavior in the inferior parietal cortex. J Neurosci 27:11028-11036.

Lu X, Ashe J (2005) Anticipatory activity in primary motor cortex codes memorized movement sequences. Neuron 45:967-973.

Mazzoni P, Bracewell RM, Barash S, Andersen RA (1996) Motor intention activity in the macaque's lateral intraparietal area. I. Dissociation of motor plan from sensory memory. J Neurophysiol 76:1439-1456.

Medendorp WP, Goltz HC, Vilis T (2006) Directional selectivity of BOLD activity in human posterior parietal cortex for memory-guided doublestep saccades. J Neurophysiol 95:1645-1655.

Mushiake H, Saito N, Sakamoto K, Itoyama Y, Tanji J (2006) Activity in the lateral prefrontal cortex reflects multiple steps of future events in action plans. Neuron 50:631-641.

Ninokura Y, Mushiake H, Tanji J (2003) Representation of the temporal order of objects in the primate lateral prefrontal cortex. J Neurophysiol 89:2869-2873.

Ohbayashi M, Ohki K, Miyashita Y (2003) Conversion of working memory to motor sequence in the monkey premotor cortex. Science 301:233-236.

Platt ML, Glimcher PW (1997) Responses of intraparietal neurons to saccadic targets and visual distractors. J Neurophysiol 78:1574-1689.

Rushworth MF, Paus T, Sipila PK (2001) Attentional systems and the organization of the human parietal cortex. J Neurosci 21:5262-5271.

Scherberger H, Andersen RA (2007) Target selection signals for arm reaching in the posterior parietal cortex. J Neurosci 27:2001-2012.

Shima K, Tanji J (1998) Both supplementary and presupplementary motor areas are crucial for the temporal organization of multiple movements. J Neurophysiol 80:3247-3260.

Shima K, Isoda M, Mushiake H, Tanji J (2007) Categorization of behavioural sequences in the prefrontal cortex. Nature 445:315-318.

Snyder LH, Batista AP, Andersen RA (1997) Coding of intention in the posterior parietal cortex. Nature 386:167-170.

Tanji J, Shima K (1994) Role for supplementary motor area cells in planning several movements ahead. Nature 371:413-416.

Todd JJ, Marois R (2004) Capacity limit of visual short-term memory in human posterior parietal cortex. Nature 428:751-754. 